PRAXIS

ecucativa

Universidad Nacional de La Pampa

Facultad de Ciencias Humanas

Instituto de Ciencias de la Educación

para la investigación interdisciplinaria

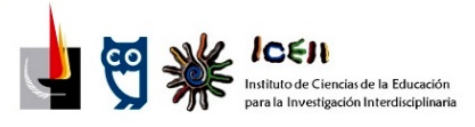

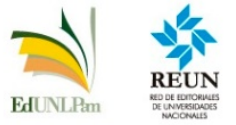

ISSN 2313-934X

SANTA ROSA, LA PAMPA, ARGENTINA

Correo electrónico: iceii@humanas.unlpam.edu.ar

Disponible en https://cerac.unlpam.edu.ar/index.php/praxis

Tensiones epistemológicas entre el conocimiento escolar y mapuche en la relación educativa intercultural. Artículo de Segundo Quintriqueo Millán, Katerin Arias-Ortega, Gerardo Muñoz Troncoso, Dykssa Saez San Martín, Karla Morales Mendoza. Praxis educativa, Vol. 26, No 1 enero - abril 2022. E - ISSN 2313-934X. pp. 1-23. https://dx.doi.org/10.19137/praxiseducativa-2022-260119

\title{
Tensiones epistemológicas entre el conocimiento escolar y mapuche en la relación educativa intercultural
}

Epistemological tensions between school and Mapuche knowledge in the intercultural educative relation

Tensões epistemológicas entre saberes escolares e Mapuche na relação educacional intercultural

\section{Segundo Quintriqueo Millán}

Universidad Católica de Temuco, Chile

squintri@uct.cl

ORCID 0000-0002-7228-4095

\section{Katerin Arias-Ortega}

Universidad Católica de Temuco, Chile

karias@uct.cl

ORCID 0000-0001-8099-0670

\section{Gerardo Muñoz Troncoso}

Universidad Austral de Chile, Chile

gerardo.munoz01@uach.cl

ORCID 0000-0002-7019-2701 


\section{Dykssa Saez San Martín}

Universidad Católica de Temuco, Chile

dsaez@uct.cl

ORCID 0000-0003-0025-6834

\section{Karla Morales Mendoza}

Universidad Católica del Maule, Chile

kmorales@ucm.cl

ORCID 0000-0001-5807-3876

Recibido: 2020-12-23 | Revisado: 2021-11-09 | Aceptado: 2021-08-18

\section{Resumen}

El artículo pretende comprender las tensiones epistemológicas en las relaciones educativas interculturales entre profesor/a mentor/a, educador/a tradicional y el estudiantado en La Araucanía. La perspectiva teórica que sustenta la discusión es la escolarización fundamentada en el currículum escolar de corte eurocéntrico occidental y la educación familiar en contexto mapuche, por lo que el estudiantado es sometido a una doble inmersión: la educación escolar y familiar. La metodología empleada es la investigación educativa, basada en una etnografía escolar y un análisis de contenido. Los principales resultados muestran la reproducción de un racismo institucionalizado en la educación escolar, una diferencia epistemológica en relación con los contenidos deseables de transmitir en la implementación de la educación intercultural y una invisibilización sistemática, tanto de los saberes indígenas como de los estudiantes y educadores tradicionales en los procesos de escolarización.

Palabras clave: escolarización y currículum escolar, conocimiento escolar y educativo mapuche, tensiones epistemológicas, pluralismo epistemológico, educación intercultural.

\section{Abstract}

The article aims to understand the tensions in the intercultural educational relationships between a mentor teacher, traditional educator and students in La Araucania. The theoretical perspective that sustains the discussion is the schooling based on the Western Eurocentric school curriculum and family education in Mapuche context, for which the students are subjected to a double immersion: school and family education. The methodology used is educational research, based on a school ethnography and content analysis. The main results show the reproductions of institutionalized racism in school education, an epistemological difference in relation to the contents desired to transmit in the implementation of intercultural education, and a systematic invisibility, both of knowledge and of traditional students and educators in schooling processes.

Keywords: schooling and school curriculum, Mapuche school and educational knowledge, epistemological tensions, epistemological pluralism, intercultural education. 
Tensiones epistemológicas entre el conocimiento escolar y mapuche en la relación educativa intercultural. | Segundo Quintriqueo Millán, Katerin Arias-Ortega, Gerardo Muñoz Troncoso, Dykssa Saez San Martín, Karla Morales Mendoza.

\section{Resumo}

0 artigo tem como objetivo compreender as tensões nas relações educacionais interculturais entre um professor mentor, um educador tradicional e alunos em La Araucania. A perspectiva teórica que sustenta a discussão é a escolarização a partir do currículo escolar eurocêntrico ocidental e da educação familiar no contexto mapuche, para a qual os alunos são submetidos a uma dupla imersão: a educação escolar e familiar. A metodologia utilizada é a pesquisa educacional, com base na etnografia escolar e na análise de conteúdo. Os principais resultados mostram as reproduções do racismo institucionalizado na educação escolar, uma diferença epistemológica em relação aos conteúdos que se deseja transmitir na implementação da educação intercultural, e uma invisibilidade sistemática, tanto dos saberes como dos alunos e educadores tradicionais nos processos de escolarização.

Palavras-chave: escolaridade e currículo escolar; escola mapuche e saberes educacionais; tensões.

\section{Introducción}

En Chile, la escolarización de estudiantes indígenas está sustentada en el currículum nacional, el que se caracteriza por ser monocultural, centralizado y homogeneizante. Está diseñado para satisfacer las necesidades de aprendizaje que se generan en el ámbito cultural, social, político y económico de la sociedad chilena. Sin embargo, al interior de las comunidades mapuches, persiste un modelo de educación familiar que permite la formación de niños, niñas y jóvenes desde una lógica propia. Es un tipo de educación apoyada en conocimientos representados en la memoria social que permite a niños, niñas y jóvenes comprender la realidad social, cultural, política y económica de un modo distinto al predominante en la sociedad chilena occidental (Quintriqueo et al., 2015). Lo anterior se constituye en tensiones epistemológicas entre el conocimiento escolar y el conocimiento educativo mapuche, que condicionan la implementación de la educación intercultural bilingüe (Quintriqueo et al., 2017).

El objetivo del presente artículo es describir las tensiones epistemológicas en la relación educativa intercultural entre el/la profesor/a mentor/a y el/la educador/a tradicional en la implementación de la educación intercultural bilingüe en el marco del currículum escolar formal, real y oculto.

\section{Escolarización y currículum escolar en contexto mapuche}

La escolarización en contexto mapuche se inscribe en el marco de un currículum escolar nacional único, el que especifica el recorrido de formación de los estudiantes en todos los niveles educativos, en las dimensiones formal, real y oculta (Quintriqueo, 2010). Esto se traduce en programas de estudios legitimados y reglamentados, según la evolución de las sociedades y los sistemas educativos escolares (Forquin, 1991; Briand y Chapoulie, 1993; Baudelot y Leclercq, 2008). Así, tanto estudiantes como profesores/as aprenden en una relación con el saber que está establecido en el marco del currículum escolar, donde el conjunto de saberes deseables de 
Tensiones epistemológicas entre el conocimiento escolar y mapuche en la relación educativa intercultural. | Segundo Quintriqueo Millán, Katerin Arias-Ortega, Gerardo Muñoz Troncoso, Dykssa Saez San Martín, Karla Morales Mendoza.

transmitir a las nuevas generaciones está definido e institucionalizado en la escuela (Perrenoud, 1994).

En el marco de la organización escolar, los objetivos, programas y planes de estudio se constituyen en un nivel de realidad abstracta (Briand y Chapoulie, 1993). En este sentido, el currículum formal es un mundo de textos y de representaciones, donde las leyes asignan los fines de la instrucción pública (Meirieu, 2007). En este marco, los programas que se desarrollan en los diversos grados y ciclos de estudios en las instituciones educativas, los métodos recomendados e impuestos, los medios de enseñanza más o menos oficiales, los itinerarios formativos y otros documentos normativos orientan la acción pedagógica (Forquin, 1991; Briand y Chapoulie, 1993). Por lo tanto, el currículum formal es construido por especialistas que no necesariamente provienen del ámbito de la educación, pero deciden, seleccionan y examinan los objetivos de aprendizaje escolar, en referencia a un/una estudiante generalmente abstracto/a (Perrenoud, 1994). Entonces, lo que dice relación con los contenidos educativos programados, planificados por los curriculistas y que deben ser realizados en el espíritu del/de la profesor/a, es lo que comprendemos como currículum formal (Perrenoud, 1994).

En el marco del currículum realizado, observamos dos aspectos centrales del currículum formal: 1) el currículum es interpretado por los/as profesores/as, quienes lo ponen en práctica en sus clases; y 2) aun cuando el currículum escolar sea completamente respetado, los contenidos no son igualmente enseñados, ya que su cobertura depende del contexto sociocultural del estudiantado (Perrenoud, 1994). Es así como la educación escolar construye las desigualdades y diferencias entre los estudiantes, en el marco de la organización escolar y las prácticas pedagógicas (Perrenoud, 2007), lo que explica, además, la desigualdad en la adquisición de conocimientos, los niveles de excelencia, el éxito escolar, educativo, profesional y económico de las nuevas generaciones (Baudelot y Leclercq, 2008; Perrenoud, 2007). Por lo tanto, las fluctuaciones y variaciones de los contenidos reales de enseñanza están ligadas a la autonomía y la subjetividad de los/as profesores/as en la interpretación de los textos, del currículum escolar y a la diversidad de las condiciones de trabajo y realidad sociocultural de los estudiantes (Baudelot y Leclercq, 2008).

En relación con las fluctuaciones y variaciones de los contenidos educativos, sostenemos que: 1) el programa de estudio es una "configuración", que es rellenada por el/la profesor/a, recurriendo a su propia relación con el saber popular, indígena, profesional y disciplinar, a su cultura, a su visión de lo que es importante y necesario para la sociedad y la cultura; 2) los/as profesores/as disminuyen selectivamente los contenidos curriculares que, a su juicio, no son significativos para el estudiantado, las familias y comunidades; 3) los manuales, cuadernos y guías de aprendizaje que utilizan los/as profesores/as se constituyen en el verdadero programa; 4) el/la profesor/a construye los pasos didácticos personales que implican una interpretación particular del programa y los contenidos del conocimiento pedagógico y disciplinario; 5) antes que el programa, el/la profesor/a se preocupa de las demandas de sus colegas, desplazando la enseñanza y el aprendizaje del estudiantado a un segundo plano; 6) la cultura, el clima 
Tensiones epistemológicas entre el conocimiento escolar y mapuche en la relación educativa intercultural. | Segundo Quintriqueo Millán, Katerin Arias-Ortega, Gerardo Muñoz Troncoso, Dykssa Saez San Martín, Karla Morales Mendoza.

pedagógico, el grado de colectividad del establecimiento influye en las exigencias y las orientaciones pedagógicas de una escuela en particular; 7) los/as profesores/as, para interpretar el currículum escolar, tienen en cuenta la composición sociológica de la comunidad escolar; 8) la enseñanza es modificada en función de los mercados escolares y profesionales, especialmente en el contexto de un currículum económico de base en una sociedad neoliberal como es el caso chileno; y 9) la comunidad local, en un sistema educativo escolar democrático, incide sobre la interpretación de la cultura escolar y las demandas de un currículum escolar contextualizado, especialmente en contextos de diversidad social y cultural (Briand y Chapoulie, 1993; Jonnaert et al., 2009; Forquin, 1991; Baudelot y Leclercq, 2008).

En el marco del currículum realizado, los profesores y estudiantes aprenden a merced de la experiencia, en una realidad compleja, múltiple, fluctuante, donde la relación educativa nunca es una expresión pura y simple como voluntad de aprender (Perrenoud, 1994). En este proceso, el/la profesor/a es parte del juego en los acontecimientos de la educación escolar, en los instrumentos de gestión institucional y en la forma de implicar a los actores del medio educativo y social (Jonnaert et al., 2009). En efecto, la experiencia de poder estructurar el recorrido de formación de cada estudiante, al punto de dejarle poco margen de libertad para construir el sentido de su vida y del lugar en el territorio, en tanto sucesión y recorrido de formación, es lo que comprendemos como currículum real (Perrenoud, 1994).

En esa perspectiva, todo currículum real está oculto porque las experiencias de los individuos no son directamente observables, porque la formación escolar es una secuencia de acontecimientos, conductas y situaciones de aprendizaje particulares (Perrenoud, 1994; Baudelot y Leclercq, 2008). Es así como una experiencia formadora compleja está hecha de múltiples momentos, a veces muy breves e intermitentes, durante largos períodos. Por consiguiente, es una realidad particularmente difícil de disociar del flujo de acontecimientos en la educación escolar. En este sentido, el currículum oculto refiere a aprendizajes que no se han planificado, pero que vienen a resultar como aprendizaje en el marco de la institución escolar (Perrenoud, 1994).

Es así como, desde una perspectiva global, los principales fines de la escuela y la educación escolar son: 1) formalizar mecanismos de aprendizaje y asegurar la posibilidad de su realización; 2) generar aprendizajes durables y sólidos, en función de objetivos y en una relación estructural con los medios utilizados; 3 ) institucionalizar el conocimiento escolar en la sociedad a través de la formación de niños y niñas como una responsabilidad exterior a la familia; y 4) legitimar social, ética, política y legalmente la estructura social, sus instituciones y las brechas sociales, económicas y educativas en el marco de la organización escolar (Meirieu, 2007). En contexto mapuche, estos fines se realizan con base en principios que orientan la escuela como institución, desde la matriz social y cultural fundada en la monoculturalidad eurocéntrica occidental como paradigma sociocultural dominante (Quintriqueo, 2010). En este sentido, la escuela y la educación se han desarrollado con base en sus hitos fundadores: colonizar y producir la inferiorización de los indígenas en América Latina y, particularmente, en la región de La 
Tensiones epistemológicas entre el conocimiento escolar y mapuche en la relación educativa intercultural. |

Segundo Quintriqueo Millán, Katerin Arias-Ortega, Gerardo Muñoz Troncoso, Dykssa Saez San Martín, Karla Morales Mendoza.

Araucanía (Quintriqueo et al., 2014). Para comprender este problema, es fundamental avanzar en el entendimiento de los saberes y conocimientos de la educación familiar mapuche.

\section{La educación familiar mapuche}

Las primeras descripciones sobre la educación mapuche se encuentran en los relatos de cronistas, principalmente misioneros que acompañaron la conquista española, escritos entre los años 1550 y comienzos de 1800. Sin embargo, desde la creación del Estado chileno en 1810, los mapuches de La Araucanía incorporaron progresivamente la educación escolar, paralelamente a su propia educación. Así, los principales efectos de la educación escolar en la vida de la población mapuche han sido: 1) la instalación de la colonialidad del ser, del poder y del hacer por parte del Estado como mecanismo de control de los grupos sociales dominantes (Battiste, 2013, 2015; Muñoz y Quintriqueo, 2019); y 2) la integración a la sociedad chilena, bajo un modelo de inclusión social básica (Poblete, 2001; Quintriqueo, 2010). Para ello, la escuela se apoyó en el monolingüismo en castellano como base para la construcción de la vergüenza étnica, el abandono del mapunzugun (lengua mapuche) y el aprendizaje de la identidad nacional chilena. Desde la lógica mapuche, la educación se sustenta en una organización social con expresiones culturales, normas de convivencia, formas de trabajo, lengua, valores educativos y una espiritualidad propia. Desde entonces, las nuevas generaciones mapuches han aprendido a convivir con una doble racionalidad educativa, entendida como la capacidad para integrar conocimientos educativos -incluso contradictorios - desde racionalidades culturales distintas (Quilaqueo et al., 2016; Quilaqueo y Quintriqueo, 2017). Así, la educación mapuche se sustenta en tres aspectos centrales: 1) una base epistémica de conocimientos educativos propios apoyada en la memoria social heredada de las colectividades mapuches históricas; 2) en la noción de relación con el saber y en las formas de organizar las preguntas sobre el conocimiento educativo mapuche; y 3) el desafío para responder a la doble racionalidad en educación, a partir de un marco de referencia que se apoya en la ecuación: pasado incorporado, contexto de acción presente y las prácticas educativas observables (Quilaqueo et al., 2016). En este sentido, la base epistémica que da cuenta de la educación familiar mapuche se apoya en la noción de acción educativa kimeltuwün (Quilaqueo et al., 2016), referido a contenidos, métodos y finalidades educativas mapuches que organizan el aprendizaje-enseñanza, según el contexto social y territorial. En el kimeltuwün, se descubre una actitud, un contenido y un procedimiento particular para comprender y explicar el medio social, cultural, natural y espiritual, con un énfasis inductivo.

La noción de relación con el saber, según Quintriqueo y Maheux (2004), Quintriqueo (2005) y Quilaqueo y Quintriqueo (2017), orientan los principales argumentos sobre la lógica de construcción social del conocimiento educativo mapuche, sustentado en los siguientes principios de formación de persona: 1) küme yamüwael ta che, actitud de respeto y estima que se debe construir en la relación entre los miembros de la familia y la red de parentesco; 2) küme rakizuam niael ta che, proceso reflexivo que canaliza el conocimiento construido; 3) kim che geael ta che, en tanto método que guía la relación aprendizaje-enseñanza sobre la base de un contenido; 4) 
Tensiones epistemológicas entre el conocimiento escolar y mapuche en la relación educativa intercultural. |

Segundo Quintriqueo Millán, Katerin Arias-Ortega, Gerardo Muñoz Troncoso, Dykssa Saez San Martín, Karla Morales Mendoza.

newen rakizuam niael ta che, referido a la relación del sujeto con el saber social, psicológico, emocional y espiritual; y 5) küme che geael ta che, como enfoque evaluativo para concluir si la persona actúa solidariamente en su comunidad.

En consecuencia, el recorrido de formación escolar y familiar de niños, niñas y adolescentes mapuches les dota de una doble racionalidad para describir, comprender y explicar el mundo mapuche y la sociedad global (Quilaqueo et al., 2016). La doble racionalidad educativa mapuche ha permitido a las familias resistir la dominación social y cultural. Por una parte, las familias han continuado la formación de sus hijos e hijas desde una racionalidad propia y, por otra, han incorporado la racionalidad escolar para integrarse a la sociedad chilena (Quilaqueo et al., 2016). Son capacidades que permiten a las personas mapuches desenvolverse adecuadamente, tanto en las familias y comunidades como en la sociedad chilena y global, desde una relación educativa intercultural.

\section{Programa de Educación Intercultural Bilingüe en Chile}

El Programa de Educación Intercultural Bilingüe en Chile (PEIB) se enmarca en un conjunto de normativas legales e institucionales. A nivel nacional, la Educación Intercultural Bilingüe (EIB) se sustenta en la Ley Indígena No 19.253 de 1993 y la Ley General de Educación de Chile (2009). A nivel internacional, a través del Convenio 169 de la Organización Internacional del Trabajo, la Declaración de las Naciones Unidas sobre los Derechos de los Pueblos Indígenas (2007) y los lineamientos de UNESCO (2008), se sostiene la necesidad de ofrecer a los pueblos indígenas una educación intercultural, ajustado a derecho, a sus propios saberes, conocimientos y lengua vernácula. En particular, la Educación Intercultural Bilingüe (EIB) en Chile surge producto de las demandas sociales, territoriales, lingüísticas y educativas de los distintos pueblos indígenas que habitan el territorio nacional, las que adquieren una mayor visibilidad desde la década de 1970 y que se concretizan como política pública desde 1996 (Ministerio de Educación de Chile [MINEDUC], 2017; Muñoz, 2021). La ElB en Chile se implementa en el marco del sistema educativo nacional y el currículum escolar formal, y no es una educación propia de los pueblos indígenas, particularmente en el caso mapuche. La EIB se ha desarrollado en tres etapas: 1) centrada en la contextualización de la educación en escuelas rurales, entre 1996 y 2000; 2) fortalecimiento del bilingüismo y la participación de las comunidades en la gestión curricular para la elaboración de Planes y Programas Propios, entre 2001 y 2009; y 3) incorporación de contenidos educativos indígenas al currículum formal, a través de la asignatura de Lengua Indígena, propiciando la revitalización lingüística, desde 2010 a la fecha (MINEDUC, 2017). La implementación de este programa se sustenta en la incorporación de las figuras de Educador/a Tradiciona/que, en el caso mapuche, se trata de un sabio de la familia y la comunidad que se incorpora a la institución escolar para apoyar la implementación de la EIB, que pone en práctica los valores y principios educativos indígenas, para la formación de los estudiantes mapuches y no mapuches. Igualmente, el/la Profesor/a Mentor/a es el/la profesional de la Educación escolar encargado/a de apoyar y monitorear el desenvolvimiento del educador tradicional en el medio escolar (Arias-Ortega y 
Quintriqueo, 2021). Siendo ambos agentes educativos actores centrales en la implementación de la EIB, desde una relación educativa intercultural.

\section{Relación educativa intercultural}

La relación educativa intercultural como marco teórico nos permite comprender la implementación de la EIB, en el que se identifican cuatro ejes: 1) el afectivo; 2) la comunicación; 3) la cooperación; y 4) el desarrollo profesional (Ver Tabla 1). Es así como la relación educativa intercultural se sostiene entre las competencias de los agentes educativos, y las condiciones estructurales y personales que favorecen la enseñanza-aprendizaje desde un enfoque educativo intercultural.

\section{Tabla 1}

Ejes y pilares para acompañar la relación educativa intercultural

\begin{tabular}{|c|c|c|}
\hline Ejes Pilares & Competencias & Condiciones \\
\hline Afectivo & $\begin{array}{l}\text { Escuchar con atención, para la apertura al } \\
\text { otro como un legítimo otro. }\end{array}$ & $\begin{array}{l}\text { Confianza y respeto mutuo, lo que implica tomar } \\
\text { tiempo para reencontrarse en actividades concretas } \\
\text { de planificación de las situaciones de aprendizaje, } \\
\text { fortaleciendo la relación educativa en perspectiva } \\
\text { intercultural. }\end{array}$ \\
\hline Comunicación & $\begin{array}{l}\text { Animar las situaciones de aprendizaje, } \\
\text { reconociendo las competencias del otro, } \\
\text { implicándose ética, política y } \\
\text { epistemológicamente en el aprendizaje } \\
\text { de los estudiantes. }\end{array}$ & $\begin{array}{l}\text { Mecanismos para compartir información, lo que } \\
\text { compromete la sistematización de saberes y } \\
\text { conocimientos educativos mapuches y escolares, para } \\
\text { el desarrollo de la situación de aprendizaje } \\
\text { intercultural. }\end{array}$ \\
\hline Cooperación & $\begin{array}{l}\text { Planificación de actividades en conjunto, } \\
\text { mediante el apoyo pedagógico en las } \\
\text { iniciativas, para concretizar el trabajo en } \\
\text { aula, desde un trabajo co-constructivo. }\end{array}$ & $\begin{array}{l}\text { Espacio y tiempo asignado que aseguren el encuentro } \\
\text { entre los agentes educativos, para la planificación y } \\
\text { desarrollo de actividades de aprendizaje en conjunto } \\
\text { entre los actores del medio educativo y social. Esto } \\
\text { implica la presencia simultánea en el aula, tanto del } \\
\text { profesor mentor como del educador tradicional u } \\
\text { otros agentes educativos de la familia y la } \\
\text { comunidad. }\end{array}$ \\
\hline Desarrollo Profesional & $\begin{array}{l}\text { Análisis reflexivo, mediante la } \\
\text { objetivación de la realidad escolar, la } \\
\text { toma de distancia crítica respecto a la } \\
\text { práctica educativa, el cuestionamiento } \\
\text { constantemente, para llegar a procesos } \\
\text { de metacognición que cuestionen } \\
\text { cualquier práctica discriminatoria y } \\
\text { práctica de racismo individual e } \\
\text { institucionalizado en la educación } \\
\text { escolar. }\end{array}$ & $\begin{array}{l}\text { Cultura pedagógica e intercultural crítica, para la } \\
\text { comprensión y explicación contextualizada del } \\
\text { sistema educativo escolar, en relación a un marco de } \\
\text { referencia pluralista, para sustentar una relación } \\
\text { educativa que promueva la gestión participativa de la } \\
\text { educación intercultural, con la implicación de la } \\
\text { familia, escuela y comunidad. En este contexto, la } \\
\text { articulación del conocimiento escolar y el } \\
\text { conocimiento educativo mapuche, permite construir } \\
\text { un recorrido de formación escolar desde un } \\
\text { pluralismo epistemológico intercultural. }\end{array}$ \\
\hline
\end{tabular}

Fuente: Arias-Ortega (2019). 
Tensiones epistemológicas entre el conocimiento escolar y mapuche en la relación educativa intercultural. | Segundo Quintriqueo Millán, Katerin Arias-Ortega, Gerardo Muñoz Troncoso, Dykssa Saez San Martín, Karla Morales Mendoza.

En la Tabla 1, Arias-Ortega (2019) señala la importancia de establecer un modelo de acompañamiento para el trabajo pedagógico desarrollado entre el/la profesor/a mentor/a y el/la educador/a tradicional, producto de los conflictos y desconocimientos que han caracterizado su interacción. Un modelo de acompañamiento debe exponer ejemplos de referencia para los/as profesores/as y los/as educadores/as tradicionales, que le permitan el desarrollo de competencias y condiciones, para asumir una postura y toma de decisiones que fortalezcan el proceso de enseñanza-aprendizaje desde un enfoque contextualizado al conocimiento sociocultural del estudiantado. La autora agrega que dichas competencias y condiciones también se constituyen en elementos pertinentes para los y las estudiantes, la escuela y la comunidad, ya que permiten una apertura a otras epistemes y, por consecuencia, justifica la implementación de la educación intercultural. Es así como el desarrollo de competencias como escuchar al otro, la planificación de actividades de aprendizaje en colaboración, el apoyo pedagógico y el análisis reflexivo permiten a los agentes educativos implementar situaciones de aprendizaje en perspectiva intercultural (Arias-Ortega, 2019; Maleyrot, 2017). Respecto a las condiciones, sostenemos que permiten implementar acciones concretas en el aula para el desarrollo de actividades de planificación en conjunto entre el/la profesor/a mentor/a y el/la educador/a tradicional, mediante el establecimiento de horarios para ello. Esto, ajustado a los instrumentos de gestión institucional de la educación escolar como el Proyecto Educativo Institucional (PEI) intercultural y los Programas de Mejoramiento Educativo (PME) (Arias-Ortega, 2019). Además, plantea el desafío de definir acciones para la sistematización del trabajo en conjunto, que promueva actividades de diálogo de saberes y conocimientos que cada uno porta, para implementar objetivos de aprendizaje comunes. Estas competencias y condiciones permiten la aplicación del conocimiento teórico y práctico en el marco de la enseñanza de la lengua y la cultura mapuche en la educación escolar, desde un compromiso de los equipos directivos, los/as profesores/as, educadores/as tradicionales, estudiantes y los/as actores/as sociales de la familia y la comunidad (Arias-Ortega, 2019).

\section{Metodología}

La metodología empleada fue la investigación educativa desde un enfoque cualitativo, para comprender y explicar las tensiones epistemológicas en la relación educativa intercultural entre el/la educador/a tradicional y el/la profesor/a mentor/a, en la implementación de la ElB (Arias-Ortega, 2019).

El estudio se realizó en tres territorialidades de La Araucanía: Wenteche, Valle Central, en la comuna de Padre Las Casas; Nagche, Vertiente Oriental de la Cordillera de Nahuelbuta, en la comuna de Galvarino; y Lafkenche, Borde Costero del Océano Pacífico, en la comuna de Saavedra. (Ver Figura 1). 


\section{Figura 1}

Área de estudio región de La Araucanía, Chile
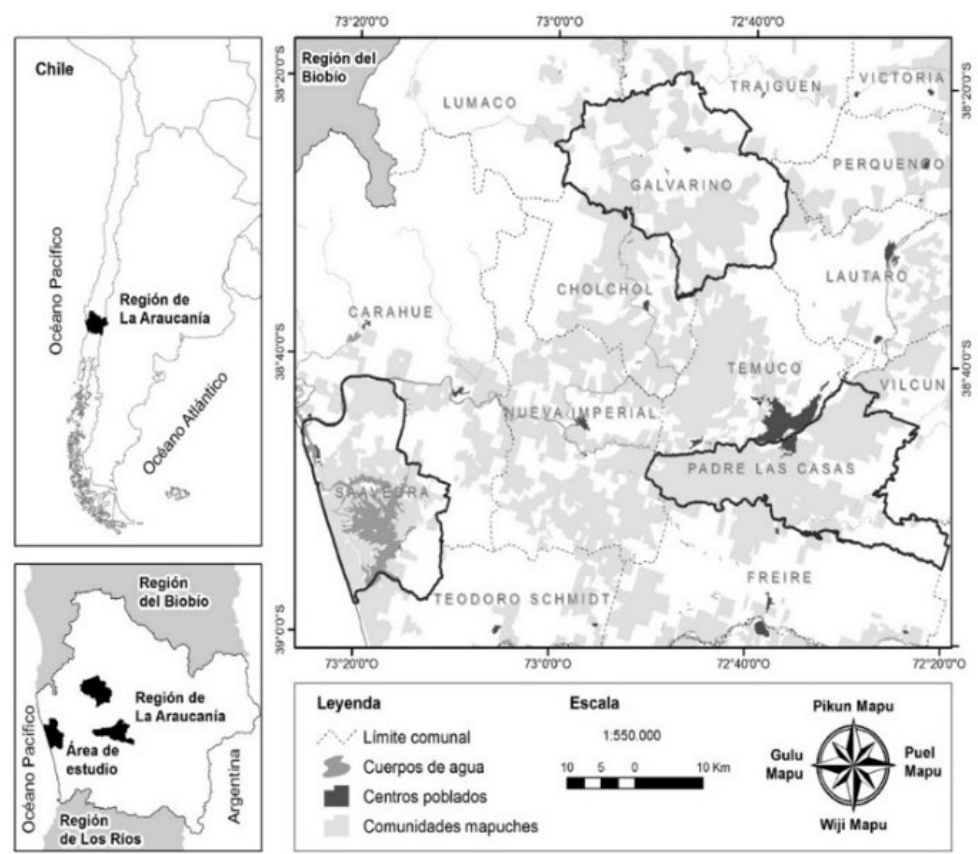

Fuente: Arias-Ortega (2019).

En cada territorialidad, se trabajó con una escuela situada en contexto de comunidades mapuches rurales. Los participantes son tres profesores/as mentores/as y tres educadores/as tradicionales, sumando un total de seis agentes educativos implicados en la implementación de la EIB. Los/as participantes fueron seleccionados/as mediante una técnica de muestreo intencional, según accesibilidad y representatividad para el/la investigador/a (Gauthier y Bourgeois, 2016).

La técnica de recolección de información fue la observación etnográfica en aula (Goetz y Le Compte, 1988), la que tiene como objetivo documentar lo explícito y latente que ocurre en la relación educativa intercultural entre el/la profesor/a mentor/a y el/la educador/a tradicional, durante la enseñanza de la lengua y la cultura mapuche en el aula. En la aplicación de la observación etnográfica, el rol de los/las investigadores/as consideró dos dimensiones principales: 1) el registro directo de las situaciones ocurridas en el contexto de estudio; y 2) la profundización de los elementos observados mediante conversaciones y entrevistas. Las técnicas y procedimientos de análisis se basó en el análisis de contenido, para describir los conceptos, dimensiones e indicadores presentes en el texto de forma explícita y latente, para construir categorías centrales en relación al objeto de estudio (Campenhoudt y Quivy, 2005). La nomenclatura de la codificación de las unidades de análisis de la etnografía realizada fue la siguiente: 1) Educador/a Tradicional de escuela Lafkenche (ETEL); 2) Profesor/a Mentor/a de escuela Lafkenche (PMEL); 3) Educador/a Tradicional de escuela Wenteche (ETEW); 4) Profesor/a Mentor/a de escuela Wenteche (PMEW); 5) Educador/a Tradicional de escuela Nagche (ETEN); 6) Profesor/a Mentor/a de escuela Nagche (PMEN); y 7) Alumno/a (AO). 
Tensiones epistemológicas entre el conocimiento escolar y mapuche en la relación educativa intercultural. | Segundo Quintriqueo Millán, Katerin Arias-Ortega, Gerardo Muñoz Troncoso, Dykssa Saez San Martín, Karla Morales Mendoza.

\section{Resultados}

Los resultados muestran la concretización de la relación educativa intercultural entre el/la profesor/a mentor/a y el/la educador/a tradicional en la implementación de la ElB, donde se observan las tensiones epistemológicas en las prácticas educativas en escuelas situadas en contexto mapuche, en las territorialidades lafkenche, nagche y wenteche.

\section{Escuela Lafkenche}

En la relación entre los agentes educativos, observamos que el/la profesor/a mentor/a y el/la educador/a tradicional, sistemáticamente, trabajan de forma paralela con dos grupos diferentes de estudiantes dentro de una misma sala de clases, sin establecer instancias de colaboración y utilizando, principalmente, el castellano en la implementación de la EIB, rechazando y negando el uso de la lengua mapuche en la situación de aprendizaje. Asimismo, durante el desarrollo de las clases, ambos agentes educativos disponen de tiempos "de manera individual" para monitorear el trabajo de los y las estudiantes. Este hecho no permite establecer una relación educativa intercultural, lo que justifica que el/la profesor/a mentor/a desarrolle la planificación de sus clases y sus guías de aprendizaje de manera individual, prescindiendo de la implicación del/de la educador/a tradicional, lo que se constata en el siguiente testimonio:

PMEL: Yo le he dicho al tío [educador tradicional] que trabajemos juntos, pero él es "flojito", no le gusta planificar. Yo le digo que haga cosas más entretenidas a los estudiantes, porque no les gusta lengua indígena. Yo tengo estas guías y planificaciones que me conseguí con un amigo, porque el tío no hace nada. Llega y se va a encerrar como dos horas al baño. (Observación etnográfica, clase $N^{\circ} 1$, escuela Lafkenche)

En el testimonio, se observa cómo el/la profesor/a mentor/a concibe al/a la educador/a tradicional como alguien incapaz de crear material educativo territorializado para la enseñanza de lengua y cultura mapuche, cuestionando la idoneidad y compromiso de este/a para la implementación de la ElB.

En ocasiones, hemos observado que el/la profesor/a mentor/a interrumpe el trabajo $\mathrm{del} / \mathrm{de}$ la educador/a tradicional, bajo el supuesto de explicar el objetivo de aprendizaje de las guías del/de la educador/a tradicional. Así, en la implementación de la ElB, observamos una ausencia de los ejes de comunicación y cooperación en la relación educativa intercultural. En el primer caso, no se reconocen las competencias del otro para motivar el aprendizaje del estudiantado. En el segundo caso, se evidencia la ausencia de planificación de las actividades en conjunto, para apoyar el trabajo pedagógico, desde una co-construcción en el aula. En este contexto de baja comunicación y cooperación se ha constatado la existencia de episodios de violencia ejercidos por el/la profesor/a hacia los y las estudiantes y al/a la educador/a tradicional, lo que se grafica a continuación:

PMEL: ¡Buuu! ¡Voy a terminar mañana contigo! ¡Buta ohhh! ¡No me gustan los trabajos feos, mira este, está todo feo!, si vamos a hacer algo lo vamos a hacer bien. ¡Si ven que esto es ancho! ¿Cómo van a hacer un árbol hacia arriba?, lo que importa es lo que está 
Tensiones epistemológicas entre el conocimiento escolar y mapuche en la relación educativa intercultural. | Segundo Quintriqueo Millán, Katerin Arias-Ortega, Gerardo Muñoz Troncoso, Dykssa Saez San Martín, Karla Morales Mendoza.

adentro, el contenido. [Le arrebata al estudiante el trabajo, se lo arruga y lo tira al basurero. Le entrega una nueva hoja y le comienza a dibujar el árbol, para que realice la actividad nuevamente]. (Observación etnográfica, clase $N^{\circ} 1$, escuela Lafkenche)

En el registro de las observaciones, constatamos que la relación educativa que establece el profesor mentor con los y las estudiantes se sustenta en una relación violenta tanto a nivel simbólico como físico. Lo anterior se expresa en las prácticas de humillación que se ejerce hacia el estudiantado al enrostrarles que su trabajo no ha sido logrado, de acuerdo con las expectativas del profesor. Otra problemática que evidenciamos tiene relación con la forma de relación educativa que establece el profesor con el educador tradicional, en el que el último asume una actitud de subalternidad, pasividad y sumisión, cuando se dirige al basurero para recuperar la actividad de aprendizaje del estudiante que el profesor ha considerado inadecuado y tirado a la basura. En estos episodios, los y las estudiantes son conscientes de la tensión existente en la relación educativa entre el profesor mentor y el educador tradicional, la que se caracteriza por la violencia verbal y no verbal en el aula. Ante estas situaciones problemáticas, los y las estudiantes reaccionan con comportamientos y actitudes de desconcentración que se expresan en conversar elevando el tono de voz, pasando por alto las instrucciones del profesor mentor y del educador tradicional, situación que incide en su desmotivación por el aprendizaje de la lengua y cultura mapuche en el aula. De este modo, la ausencia de comunicación entre el profesor mentorestudiantes-educador tradicional genera un clima de relaciones negativas para el aprendizaje, afectando el clima de aula. Asimismo, el silencio de los y las estudiantes ante las interrogantes de los agentes educativos se sanciona con la aplicación de evaluaciones como una forma de "castigo", impedimentos para salir a los recreos y amenazas de llamar a los padres y madres para denunciar sus actitudes, lo que se grafica a continuación:

PMEL: ¡Buta ohhhh! ¿Qué pasa hoy? ¡Están todos flojos! ¡Querían recreo y ni siquiera han terminado! ¿Cómo es eso? [Ante estos reclamos, los y las estudiantes se quedan en silencio].

PMEL: ¡Ya apúrense, ohhh! ¿Cómo tan flojos y lentos? ¿Buta, ohhh, qué cosa no entienden! ¿Cómo tan lentos? ¿Cuántas veces quieren que les repita las mismas cosas a ustedes? Los sustantivos propios van con mayúscula. ¿Por qué me escriben con minúscula los sustantivos propios? ¡No se pueden olvidar de eso!, porque yo no soy una grabadora [El profesor mentor utiliza un tono de voz elevado durante la interacción con los y las estudiantes]. (Observación etnográfica, clase №2, escuela Lafkenche)

En este tipo de situaciones de aprendizaje, observamos la ausencia del eje afectivo de la relación educativa intercultural asociado a una falta de confianza y respeto mutuo en el desarrollo de las situaciones de aprendizaje. Es así como, lejos de estimular y motivar un clima propicio para el aprendizaje de la lengua y la cultura mapuche, se genera una relación educativa remarcada por los desacuerdos entre el profesor mentor-estudiantes-educador tradicional sustentada en la intimidación y la sanción. Este desacuerdo entre los agentes educativos evidencia una tensión epistemológica en relación con la forma de comprender el acto de aprender y enseñar, según la 
Tensiones epistemológicas entre el conocimiento escolar y mapuche en la relación educativa intercultural. | Segundo Quintriqueo Millán, Katerin Arias-Ortega, Gerardo Muñoz Troncoso, Dykssa Saez San Martín, Karla Morales Mendoza.

racionalidad de la educación escolar y la educación familiar mapuche. Esto también es desarrollado por el educador tradicional, lo que se ilustra en el siguiente registro:

ETEL: ¡A ver! ¡A ver! ¡Un poquito de atención por favor! ¿Qué se hace al comenzar en la mañana, a ver? ¡Ajkütuymün! ¿Nos saludamos hoy día o no? ¡A ver! [para llamar la atención de los y las estudiantes, aplaude y eleva el tono de voz. Estos/as ignoran las palabras del educador tradicional y continúan organizando sus grupos de trabajo y conversando entre ellos]

ETEL: ¡Escuchen, por favor! [gritando, solicita la atención de los estudiantes] ¡Saluden en mapuche zugun! (Observación etnográfica, clase N² 2, escuela Lafkenche)

En esta dinámica de relación educativa intercultural, el educador tradicional asume una actitud de desesperación que lo altera y distrae en su práctica educativa, al no tener respuesta de los estudiantes. Esto genera en él ansiedad, intentando que los estudiantes saluden en mapunzugun, sin tomar consciencia que él mismo no lo hace. El profesor mentor, ante esta situación, interviene y expresa el deseo de que el concepto de chaliwün (saludo) sea bien aprendido por los y las estudiantes. Para ello, solicita al educador tradicional y a la investigadora recrear una situación de chaliwün. Durante esta actividad, se constata la apertura y disposición que asumen los y las estudiantes para aprender la lengua mapuche mapunzugun. Asimismo, la atención y concentración en la actividad ilustra el compromiso de los y las estudiantes por el aprendizaje, al observar y escuchar el uso del mapunzugun en un clima de respeto. Esto es valorado por el profesor mentor, quien menciona:

PMEL: con esto que los tíos [educador tradicional e investigadora] hicieron, quiero que ustedes vean que es linda la pronunciación del mapunzugun. Cuando uno aprende la lengua mapuche, agilizamos nuestro cerebro, aprendemos otro idioma. Esto lo tenemos que llevar en el corazón, porque el mapunzugun nos ayuda para aprender el inglés, la pronunciación. jA mí me encantaría aprender! Por eso el tío dijo ya, vamos a trabajar el grafemario unificado. (Observación etnográfica, clase №3, escuela Lafkenche)

En relación con las observaciones de aula, constatamos un episodio fortuito de apertura del profesor mentor hacia la lengua y cultura mapuche. Sin embargo, persiste una negación del profesor mentor, quien "no acepta" los contenidos educativos mapuches que propone incorporar el educador tradicional a las prácticas educativas escolares, lo que se expresa en una crítica constante al material educativo preparado por el educador tradicional. Esto es acompañado por una búsqueda constante de simplificar y reducir el aprendizaje del mapunzugun a elementos técnicos y parcializados de la lengua. Es así como constatamos que existe un desacuerdo permanente entre el profesor mentor y el educador tradicional, lo que tensiona epistemológicamente la relación educativa intercultural, porque los contenidos educativos deseables de enseñar en la escuela están en constante contraposición entre la racionalidad de la educación escolar y la educación familiar mapuche. Al respecto, en el aula, observamos lo siguiente: 
Tensiones epistemológicas entre el conocimiento escolar y mapuche en la relación educativa intercultural. | Segundo Quintriqueo Millán, Katerin Arias-Ortega, Gerardo Muñoz Troncoso, Dykssa Saez San Martín, Karla Morales Mendoza.

PMEL: Tío [educador tradicional], trabajemos con el abecedario más fácil, con el que se escriba la palabra tal como se oye, para que los estudiantes aprendan.

ETEL: Ya, trabajemos con el [grafemario] unificado, yo los manejo todos, pero para usted y los niños como no saben mucho se les hará más fácil con este.

PMEL: Ya, ahora pasemos materia, porque si no pasamos materia los niños se desordenan. Ya, chiquillos, ahora vamos a conocer un abecedario, ¿Cuál es el abecedario en español? Aos: $A, b, c, d, e, f, g .$. (los y las estudiantes continúan diciendo en voz alta el abecedario) PMEL: Bien, pero ahora vamos a conocer el abecedario en mapuche, cópienlo en su cuaderno. El tío lo va a escribir en la pizarra. (Observación etnográfica, clase № 3, escuela Lafkenche)

Si bien existe una reducción del mapunzugun a un componente lingüístico, se constata una instancia de trabajo conjunto entre el profesor mentor y educador tradicional, situación que sorprende a este último, quien le manifiesta a la investigadora que: "esta es la primera ocasión (después de cuatro años de actividades en común) que he visto al profesor mentor 'implicarse' de manera efectiva en el aula, mediante el desarrollo de una actividad que yo propongo" (ETEL, Clase $N^{\circ} 3$, escuela Lafkenche). Es así como constatamos que la valorización explícita de los contenidos, métodos y finalidades educativas de la educación familiar mapuche en la escuela, como es el chaliwün, cambia favorablemente la actitud y el interés del estudiantado por aprender según los principios de la pedagogía indígena. No obstante, esta es una situación aislada y no cambia la percepción del profesor mentor y se refleja en un rechazo sistemático a colaboración con el educador tradicional. Esto queda en evidencia en el siguiente registro:

PMEL: [Frente a los/as estudiantes y con un tono de voz elevado] ¡Bucha, tío! Ese es otro problema suyo, todas las guías se las lleva, no les deja nada a los estudiantes para que estudien en la casa. Además, sus guías están con muchas faltas de ortografía, usted solo copia de internet la información y la copia mal. Siempre le he dicho que lo importante es el contenido. ¡Bucha, entonces no se po', que van a aprender los chiquillos! (Observación etnográfica, clase $N^{\circ} 2$, escuela Lafkenche)

De esta forma, se genera un bajo desarrollo profesional debido a una ausencia de reflexión y toma de distancia crítica respecto de la práctica educativa, tanto del educador tradicional como del profesor mentor, asociado a una falta de metacognición que cuestione las prácticas de violencia mutua y el desinterés por ofrecer una situación de aprendizaje pertinente y significativa para el estudiantado.

\section{Escuela Wenteche}

La relación educativa intercultural entre el profesor mentor y el educador tradicional en la escuela Wenteche se caracteriza por una apertura a la colaboración. De esta manera, se generan espacios de participación conjunta referida a la incorporación del saludo en mapunzugun con los y las estudiantes, en el que ambos agentes educativos participan activamente. En este contexto, para las y los estudiantes, es habitual realizar esta práctica en cada una de las clases de lengua 
Tensiones epistemológicas entre el conocimiento escolar y mapuche en la relación educativa intercultural. |

Segundo Quintriqueo Millán, Katerin Arias-Ortega, Gerardo Muñoz Troncoso, Dykssa Saez San Martín, Karla Morales Mendoza.

indígena, donde observamos actitudes cordiales entre educador tradicional-estudiantes-profesor mentor.

Constatamos que, en la relación educativa intercultural, un eje central es la cooperación en la construcción de material educativo para la enseñanza del mapunzugun, prescindiendo de los textos escolares entregado por el MINEDUC, lo que permite la territorialización de la EIB. Sin embargo, en las observaciones realizadas, hemos constatado que el profesor mentor toma la iniciativa de escribir vocabulario en el pizarrón; no obstante, el educador tradicional asume una actitud de supervisor, haciéndole notar constantemente sus errores en la escritura en mapunzugun, lo que tensiona la relación entre ambos agentes educativos. En efecto, la presencia de la racionalidad educativa escolar y de la educación familiar mapuche en la escuela es la causa principal de las tensiones epistemológicas entre el profesor mentor y el educador tradicional en la relación educativa intercultural. Pensamos que no se trata de una tensión de voluntades personales o profesionales, sino, más bien, un trasfondo epistemológico que define el carácter de la educación, según la cosmovisión eurocéntrica occidental y mapuche en particular. Lo anterior se expresa en el siguiente testimonio:

ETEW: profesor, eso está malo, no se escribe así [el educador tradicional se dirige al profesor mentor para hacerle saber que una indicación que ha dado a los estudiantes está equivocada].

PMEW: Ya, bórrelo nomás, si considera que está malo arréglelo, usted es la que sabe [EI profesor mentor expresa su molestia a través del lenguaje no verbal, asociado al movimiento de cejas y hombros]. (Observación etnográfica, clase $N^{\circ} 4$, escuela Wenteche)

De acuerdo con el registro, constatamos la ausencia del eje afectivo y de comunicación en la relación educativa intercultural entre el profesor mentor y el educador tradicional, lo que se evidencia cuando el profesor mentor se molesta por las constantes correcciones que realiza el educador tradicional respecto del uso oral y escrito del mapunzugun. Si bien se constata el desarrollo de actividades de aprendizaje en mapunzugun, en general, los contenidos educativos mapuches son enseñados en castellano, centrado principalmente en la transcripción de textos escritos en mapunzugun en el pizarrón, careciendo de su uso práctico.

Constatamos que la incorporación sistemática de contenidos educativos mapuches refiere al gübam (consejo), el gijatun (ceremonia socio-religiosa mapuche), meli folil küpan (raíces de ascendencia materna y paterna) y las diferencias que existen en las distintas prácticas socioculturales mapuches, según territorialidad. Al educador tradicional le corresponde la explicación y profundización de dichos contenidos, exponiendo su significado e importancia para la cultura mapuche. Un ejemplo de ello es cuando trabajan los contenidos del "üy che" (nombre de las personas) y "üy mapu" (nombre de los distintos territorios). Es lo que observamos en el siguiente extracto de una clase:

ETEW: [...] como los no mapuches no sabían escribir nuestros apellidos, lo escribían como lo escuchaban. Entonces, por eso es que está como distorsionado. Por ejemplo, ahí dice 
Tensiones epistemológicas entre el conocimiento escolar y mapuche en la relación educativa intercultural. | Segundo Quintriqueo Millán, Katerin Arias-Ortega, Gerardo Muñoz Troncoso, Dykssa Saez San Martín, Karla Morales Mendoza.

Llankafilo. ¡Qué lindo apellido! [expresa emoción]. ¿Este es un nombre de che o de üy mapu? ¿Chem üy? ¿Üy che o üy mapu?

Aos: Üy che

ETEW: ¡Muy bien!, porque es el apellido de Jonás, aquí hay dos palabras juntas. [...] Pero

Llanka es una cosa y filu es otra cosa. ¿Qué será Llanka? ¿Chem am ta Llanka? ¿Lo han escuchado? ¿Llankatu, tampoco? o ¿Münarpa Ilankatu, niekefuy ni ñaña? Las ñañitas siempre utilizan mucho /lankatu. ¿Lo han escuchado? ¿Qué será, a ver? Por ahí tenemos un Ilankatu que tiene la ñaña. ¿Qué será?

Ao: es como una pulsera, es como una cosita...

ETEW: Ahí vamos avanzando, /lanka viene de Ilankatu. El /lankatu son como las perlas, son como las perlas preciosas esas que brillan. ¿Qué son, entonces, los /lankatu?

Aos: Las perlas.

ETEW: Las perlas, cierto, ¿Y filu qué será? ¿Qué será filu?

Ao: Serpiente.

ETEW: Serpiente, culebra, cierto, serpiente. ¿Y entonces qué será si los juntamos? ¿Qué nos dirá? ¿Chem piley?

Ao: Las perlas de las serpientes.

ETEW: Es al revés, una serpiente preciosa, brillante, que brilla entonces como...

Aos: las perlas. (Observación etnográfica, clase $N^{\circ} 2$, escuela Wenteche)

De acuerdo con el registro, podemos constatar que la enseñanza de la toponimia y el nombre de las personas en el conocimiento mapuche son desarrollados principalmente mediante la descomposición de las partículas lingüísticas que los componen, para develar su sentido y significado en la sociedad y cultura mapuche. Constatamos que la implementación de la educación intercultural y la relación educativa intercultural, entre el profesor mentor y el educador tradicional, se enfrentan al desafío de incorporar conocimientos educativos mapuches sustentados en la episteme propia, lo que no siempre es coherente con los contenidos y métodos educativos escolares. Esto significa que, para dar sentido y significado a los contenidos educativos mapuches en la educación, es necesario recurrir a la memoria social y al aprendizaje de experiencias, según la educación familiar mapuche. Por el contrario, en la escuela se concibe una enseñanza y aprendizaje de los contenidos educativos en abstracto, en la sala y desde la pizarra.

En esta misma dinámica, se evidencian ejemplos de contenidos mapuches abordados en relación con las nociones de tiempo y espacio en la cultura mapuche, proceso en el que el educador tradicional asocia a los diferentes tipos de actividades que pueden desarrollarse en su territorio. Para ello, ejemplifica con las actividades que se desarrollan en la comunidad, los tipos y tamaños de las ruka (casas). En este mismo espacio, se observa que el profesor mentor ejemplifica estos tiempos en función de procesos y hechos históricos del pueblo mapuche, asociando, por ejemplo, al tiempo pasado, a la llegada de los no mapuches al territorio de La Araucanía. Aquí, emerge la conquista y la colonización como otro punto de desacuerdo 
Tensiones epistemológicas entre el conocimiento escolar y mapuche en la relación educativa intercultural. | Segundo Quintriqueo Millán, Katerin Arias-Ortega, Gerardo Muñoz Troncoso, Dykssa Saez San Martín, Karla Morales Mendoza.

epistemológico para lograr una comprensión de la realidad sociocultural en la actualidad. Lo anterior se observa en el siguiente registro:

Se observa que el educador tradicional se acerca a un estudiante quien dice que el apellido de su abuela es Soruco. Sin embargo, el educador tradicional le insiste que ese no es el apellido de su abuela, que averigüe bien. El profesor mentor, al darse cuenta de esta situación, le dice al educador que lo deje así nomás, que no insista en el apellido del estudiante, que, si la mamá le había dado ese apellido en la casa, ese debe de ser. El educador tradicional le menciona a la investigadora que ella ha vivido todo el tiempo en la comunidad y que sabe que el apellido del estudiante no es Soruco, sino Kalfumañ. [...] El educador tradicional insiste que revisen el registro de los libros del colegio, que ahí deben estar los apellidos correctos. (Observación etnográfica, clase $N^{\circ} 2$, escuela Wenteche)

De esta manera, observamos, en la clase, que el educador tradicional discute con insistencia con el estudiante (menor de 12 años) sobre un eventual cambio de apellido en su familia. El profesor mentor, ante esta situación, le llama la atención al educador tradicional y, con ello, busca finalizar con la controversia. Así, la intervención del profesor mentor permite evitar la generación de un conflicto psicosocial en sus estudiantes, durante la enseñanza del mapunzugun.

\section{Escuela Nagche}

En la relación educativa intercultural entre el profesor mentor y el educador tradicional, observamos que practican el saludo en mapunzugun sistemáticamente y quienes olvidan parte del saludo reciben la cooperación mediante palabras clave que les permite realizar la práctica del chalin (saludo). Es lo que observamos en el siguiente extracto de una clase:

ETEN: Mari mari pu püchike che (Buenos días, niños)

Aos: Mari mari kimeltuchefe (Buenos días, profesor/a)

ETEN: ¿Chumleymün? (¿Cómo están?)

Aos: Kümelkalen ¿eymi kay? (Estoy bien. ¿Y usted?)

ETEN: Kümelkalen, feley (Estoy bien)

PMEN: Mari mariSebastián (Buen día, Sebastián)

Ao: Inche tañi üy Sebastián Guzmán pigen, tañi chijkatuwe La Piedra Pigey, tañi waria Galvarino pigey, tañi ñuke küpan Conejeros pigey, tañi chaw küpan Guzmán, pigey (Mi nombre es Sebastián Conejeros, mi escuela es La Piedra y mi ciudad es Galvarino, mi ascendencia materna es Conejeros y mi ascendencia paterna es Guzmán)

ETEN: ¿Tami lof? (¿Y tu comunidad?)

Ao: Tañi lof Xankilko grande pigey. (Mi comunidad es Tranquilco Grande). (Observación etnográfica, clase $N^{\circ} 2$, escuela Nagche)

En clases, generalmente, el educador tradicional es quien introduce la explicación del contenido sobre la enseñanza-aprendizaje, por lo que se traducen palabras del mapunzugunal castellano para ser repetido de forma oral y luego escritas por las y los estudiantes. Estas actividades son complementadas con la construcción y memorización de diálogos en 
Tensiones epistemológicas entre el conocimiento escolar y mapuche en la relación educativa intercultural. |

Segundo Quintriqueo Millán, Katerin Arias-Ortega, Gerardo Muñoz Troncoso, Dykssa Saez San Martín, Karla Morales Mendoza.

mapunzugun, lo que permite al profesor mentor y al educador tradicional evaluar los aprendizajes adquiridos por el estudiantado sobre la lengua y la cultura mapuche, desde la lógica de la cultura escolar.

En la implementación de la ElB, el educador tradicional, generalmente, se ubica en un rincón de la sala de clases revisando su teléfono celular, mientras es el profesor mentor quien asume el liderazgo de la situación de aprendizaje. Aquí, la actitud del educador tradicional puede ser interpretada de muchas formas, pero pensamos que algunas causas son: 1) un desinterés por la lógica de la educación escolar para aprender y enseñar contenidos educativos mapuches, por considerar que está en contraposición a la educación familiar mapuche; 2) una ausencia de competencias didácticas y pedagógicas para estimular el aprendizaje de los estudiantes mapuches y no mapuches; y 3 ) un desconocimiento de los contenidos, métodos y finalidades educativas mapuches para apoyar la implementación de la ElB. Ello lo observamos en el siguiente extracto de una clase: "PMEN: A ver si el educador nos ayuda. La intención es que participe en la clase y no continúe mirando su celular. ETEN: No... no les puedo ayudar, porque cantan muy mal, cantan mal la canción (Observación etnográfica, clase Nº 1, escuela Nagche)".

De acuerdo con la observación, constatamos que, en ocasiones, el educador tradicional se niega explícitamente a participar en clases, bajo la justificación de que la pronunciación de las y los estudiantes no es adecuada. De esta forma, se observa en el educador tradicional una escasa motivación por favorecer el aprendizaje de sus estudiantes, dando cuenta, además, de una baja tolerancia a la frustración frente a las dificultades de aprendizaje. Este tipo de actitudes genera una relación educativa tensionada con el profesor mentor, y limita la cooperación para la implementación de la educación intercultural.

\section{Discusión}

De acuerdo con los resultados de la investigación, hemos constatado la existencia de una relación educativa intercultural tensionada epistemológicamente entre el/la profesor/a mentor/a y el/la educador/a tradicional. Pensamos que las principales causas de este problema son: 1) todos/as los/as educadores/as tradicionales que participan del estudio han sido escolarizados/as en el marco de una educación escolar eurocéntrica y monocultural, por lo que limita concebir el aprendizaje y la enseñanza de contenidos educativos mapuche en la implementación de la ElB; 2) asimismo, los/as educadores/as tradicionales han recibido una educación familiar, desde principios de la pedagogía y la educación propia, que los dota de una racionalidad para comprender las situaciones de aprendizaje, según la cosmovisión mapuche; y 3) la incorporación de saberes y conocimientos educativos mapuches se enmarca en una distancia entre la racionalidad de la educación escolar y la educación familiar mapuche, lo que está a la base de la tensión epistemológica en la relación educativa intercultural entre el/la profesor/a mentor/a y el/la educador/a tradicional. El conjunto de causas identificadas en la tensión epistemológica, en la relación educativa intercultural, limitan la calidad de los procesos de enseñanza y aprendizaje para estudiantes indígenas y no indígenas, en el marco de la implementación de la EIB. 
Tensiones epistemológicas entre el conocimiento escolar y mapuche en la relación educativa intercultural. | Segundo Quintriqueo Millán, Katerin Arias-Ortega, Gerardo Muñoz Troncoso, Dykssa Saez San Martín, Karla Morales Mendoza.

Igualmente, hemos constatado la existencia de una desterritorialización de los contenidos educativos mapuches incorporados en la intervención educativa intercultural, debido a que el currículum escolar en Chile es único y centralizado, lo que aumenta la desigualdad educativa en contexto indígena. De esta manera, en La Araucanía, la escolarización está instituida exclusivamente en el marco curricular nacional, lo que constituye un problema para la implementación de la educación intercultural. Lo anterior, debido a que la escolarización promueve entre los y las estudiantes conocimientos y discursos del mundo académico alienado por la monoculturalidad eurocéntrica occidental y al monolingüismo en castellano (Quintriqueo y Quilaqueo, 2019). Esto explicaría el hecho de que, en las escuelas estudiadas, hemos constatado como patrón común la enseñanza de la lengua mapuche sustentada en la traducción de palabras e ideas al castellano. Estos hallazgos concuerdan con los estudios de Quidel (2011), Loncon et al. (2016) y MINEDUC (2018), quienes constatan que la enseñanza del mapunzugun en la escuela se realiza desde la estructura gramatical del castellano, lo que permite mantener el arraigo colonial en la implementación de la EIB en contexto mapuche (Webb y Radcliffe, 2013; Muñoz y Quintriqueo, 2019).

En esa perspectiva, en contexto mapuche, existe un discurso histórico del profesorado y de educadores/as tradicionales que niegan la incorporación de saberes y conocimientos mapuches en la educación escolar para favorecer el aprendizaje de los contenidos disciplinarios del currículum escolar. Este es un problema complejo de superar, principalmente a nivel epistemológico, puesto que es necesario visualizar los compromisos que aparecen entre las reglas y normas de validación de cada grupo social en la construcción y realización del currículum en la escuela (Maheux et al., 2020). Sin embargo, sostenemos que es posible superar la descalificación de los saberes y conocimientos educativos mapuches sobre la base de un pluralismo epistemológico para iniciar una escolarización dialógica y crítica, basada en la relación de saberes y en un proceso de descolonización de la escolarización en contextos indígenas (Maheux et al., 2020). Es decir, en el que los puntos de divergencias y acuerdos entre opciones epistemológicas sean posibles de articular en los Proyectos Educativos de las escuelas que implementan la ElB.

En esa perspectiva, la relación educativa intercultural está condicionada por el currículum formal que establece los contenidos, métodos y finalidades educativas propias para la enseñanza de la lengua y cultura mapuche. No obstante, en las observaciones, hemos constatado que no existe un proceso de planificación curricular coherente a los contenidos, métodos y finalidades educativas propuestas en el currículum de la asignatura de lengua indígena, los que, a su vez, no se relacionan con las particularidades del conocimiento mapuche según territorialidades. Desde el currículum real, estas problemáticas propician que tanto profesores/as mentores/as como educadores/as tradicionales prescindan del uso del currículum formal en la enseñanza del mapunzugun, siendo ellos/as quienes definen de manera circunstancial los contenidos y métodos educativos a incorporar, en general, carente de una planificación cooperativa que permita construir una relación educativa intercultural. 
En el plano del currículum oculto, constatamos en la relación educativa intercultural una alta recurrencia de violencia explícita e implícita hacia el estudiantado y educadores/as tradicionales. Son violencias ejercidas hacia sujetos mapuches, asociado a expresiones de racismo institucionalizado, discriminación y prejuicios que construyen una minorización y subalternización de lo indígena (Arias-Ortega, 2019). En este escenario, en la relación educativa intercultural, los/as profesores/as mentores/as y educadores/as tradicionales desconocen u omiten su propia responsabilidad social, política, ética y epistemológica en relación con el silenciamiento de las y los estudiantes indígenas (Foley, 2004; Novaro, 2016).

\section{Conclusiones}

Concluimos que las condiciones de implementación actual de la EIB en contexto mapuche limitan la relación educativa intercultural entre profesores/as mentores/as-educadores/as tradicionales-estudiantes. Asimismo, contribuyen a la reconstrucción cíclica de prejuicios étnicos y desigualdades educativas, asociado a una baja cobertura y progresión curricular. En consecuencia, el racismo institucionalizado, el monoculturalismo eurocéntrico occidental y el arraigo colonial limitan la calidad de los aprendizajes y la implementación de la EIB. Tensiones que se relacionan con: 1) la negación de reconstruir la historia familiar mediante la implementación de la EIB; 2) el intento sistemático de estudiar el conocimiento indígena en el orden epistémico del conocimiento eurocéntrico occidental; 3) la enseñanza del mapunzugun traducido a la estructura gramatical de la lengua castellana; y 4) la noción racista del "otro indígena" como conflictos que impiden el entendimiento adecuado del saber y conocimiento educativo mapuche en la educación escolar. Finalmente, constatamos que existe una relación educativa de carácter canónico, en la que el/la profesor/a sostiene la hegemonía del saber y del poder, asumiendo un autoritarismo y unilateralidad en los procesos de enseñanza y aprendizaje. En ese sentido, existe una ausencia de relación educativa intercultural entre los actores del medio educativo y social, porque no se implementan procesos educativos interculturales, según los ejes y pilares que la deberían constituir y sostener en la implementación de la EIB.

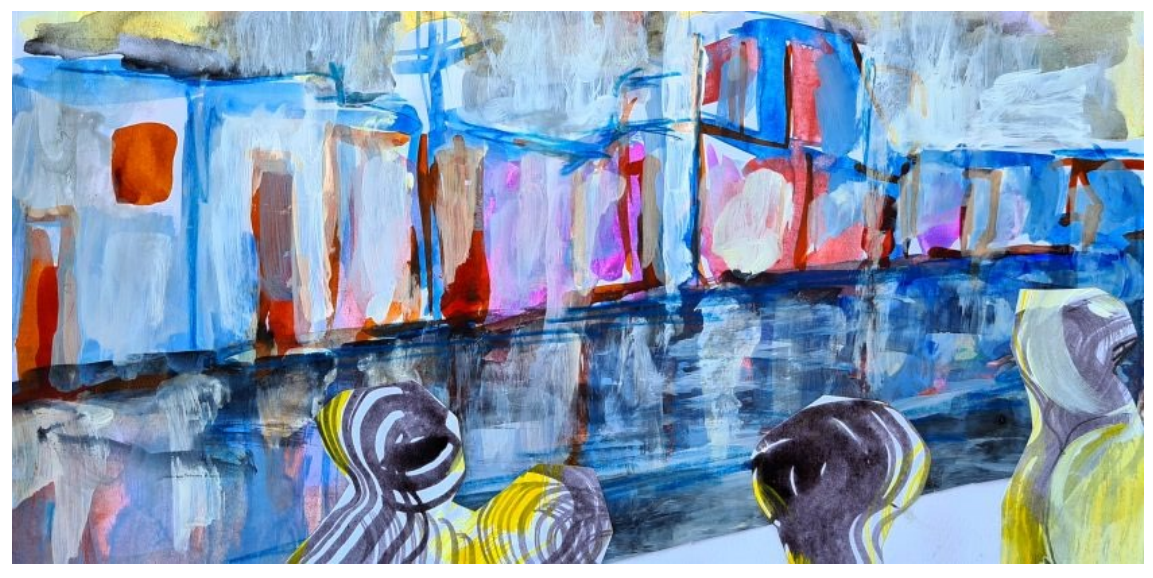

Humanos y más, acrílico y tinta sobre papel. Ana María Martin 


\section{Agradecimiento}

Proyectos FONDECYT Regular 1181531, FONDECYT Iniciación 11200306 y 11191041, FONDEF ID21110187.

\section{Bibliografía}

Arias-Ortega, K. (2019). Relación educativa entre el profesor mentor y el educador tradicional en la educación intercultural[Tesis Doctoral]. Universidad Católica de Temuco.

Arias-Ortega, K., y Quintriqueo, S. (2021). Tensiones epistemológicas en la implementación de la Educación Intercultural Bilingüe. Ensaio Avaliação e Políticas Públicas em Educação 29(2) 1-19. DOI: 10.1590/s010440362020002802249

Baudelot, C. y Leclercq, F. (2008). Los efectos de la educación. Del estante. Briand, J. P. y Chapoulie, J. M. (1993). L'institution scolaire et la scolarisation: une perspective d'ensemble. Revue Française de Sociologie, 34(1), 3-42. https://www.persee.fr/doc/rfsoc_0035-

2969_1993_num_34_1_4217

Campenhoudt, L. V. y Quivy, R. (2005). Manual de Investigação em Ciências Sociais. Gradiva-Publicações. Foley, D. E. (2004). El indígena silencioso como una producción cultural. Cuadernos de Antropología Social, (19). https://www.redalyc.org/pdf/1809/180913911002.pdf Forquin, J. C. (1991). Savoirs scolaires, contraintes didactiques et enjeux sociaux. Sociologie et sociétés, 23(1), 25-39.

Gauthier, B. y Bourgeois, I. (2016). Recherche sociale de la problématique à la collecte des données. Presses de l'Université du Québec.

Houssaye, J. (1993). La pédagogie, une encyclopédie pour aujourd'hui. ESF. Jonnaert, P., Ettayebi, M. y Defise, R. (2009). Curriculum et compétences: un cadre opérationnel. De Boeck Supérieur.

Loncon, E., Castillo, S. y Soto, J. (2016). Barreras a la interculturalidad en el sistema educativo chileno. Informe final. UNICEF y MINEDUC.

Maheux, G., Pellerin, G., Quintriqueo, S. y Bacon, L. (2020). La décolonisation de la scolarisation des jeunes Inuit et des Premières Nations: Sans et défis. Presses de l'Université du Québec.

Maleyrot, É. (2017). Le travail partagé dans le dispositif «plus de maîtres que de classes»: contrastes des formes de collaboration et des dynamiques professionnelles. Les Sciences de l'education-Pour l'Ere nouvelle, 50(3), 31-55. https://www.cairn.info/revue-les-sciences-de-I-education-pour-I-ere-nouvelle-20173-page-31.htm Meirieu, P. (2007). En la escuela hoy. Octaedro.

MINEDUC. (2009). Programa de Educación Intercultural Bilingüe. Consulta a los pueblos indígenas informe de resultados: función docente del educador(a) tradicional para la implementación del sector de lengua indígena. Ministerio de Educación.

MINEDUC. (2018). Prácticas pedagógicas interculturales: reflexiones, experiencias y posibilidades desde el aula. Salesianos Impresores S.A.

Morales, C. (2015). Nociones y principios de la educación intercultural presentes en prácticas pedagógicas realizadas en contexto de interacción juvenil rural de la región Metropolitana (Chile). Diálogo Andino, 47-5970. http://dx.doi.org/10.4067/s0719-26812015000200007 
Tensiones epistemológicas entre el conocimiento escolar y mapuche en la relación educativa intercultural. |

Segundo Quintriqueo Millán, Katerin Arias-Ortega, Gerardo Muñoz Troncoso, Dykssa Saez San Martín, Karla Morales Mendoza.

Muñoz, G. (2021). Educación familiar e intercultural en contexto mapuche: hacia una articulación educativa en perspectiva decolonial. Estudios Pedagógicos, 47(1), 391-407. https://doi.org/10.4067/S0718-

07052021000100391

Muñoz, G. y Quintriqueo, S. (2019). Escolarización socio-histórica en contexto mapuche: implicancias educativas, sociales y culturales en perspectiva intercultural. Educação e Sociedade, 40, e0190756. http://dx.doi.org/10.1590/es0101-73302019190756.

Novaro, G. (2016). ¿Desafíos interculturales o una interculturalidad desafiada? Experiencias en escuelas con población migrante boliviana en Argentina. Temas de Educación, 27(2), 381-390.

https://revistas.userena.cl/index.php/teduacion/article/view/683

Novaro, G., Padawer, A. y Borton, L. (2017). Interculturalidad y Educación en Argentina desde una Perspectiva Comparativa. Educação \& Realidade, 42(3), 939-958. http://dx.doi.org/10.1590/2175-623672321

Perrenoud, P. (1994). Le travail sur l'habitus dans la formation des enseignants: analyse des pratiques et prises de conscience. Faculté de psychologie et de sciences de l'éducation: Service de la recherche sociologique.

Perrenoud, P. (2007). Pedagogía diferenciada: de las intenciones a la acción. Editorial popular. Poblete, M. (2001). Comunidades mapuches de Panguipulli y educación: las primeras décadas del siglo XX. Revista Austral de Ciencias Sociales, 5, 15-27. https://doi.org/10.4206/rev.austral.cienc.soc.2001.n5-02 Quidel, J. (2011). Las relaciones interétnicas desde la perspectiva mapuche. Universidad Católica de Temuco. Quilaqueo, D. y Quintriqueo, S. (2017). Métodos educativos Mapuches: retos de la doble racionalidad educativa. Aportes para un enfoque de educación intercultural. Universidad Católica de Temuco.

Quilaqueo, D., Quintriqueo, S., Riquelme, E. y Loncón, E. (2016). Educación mapuche y educación escolar en La Araucanía: ¿doble racionalidad educativa? Cuadernos de Pesquisa, 46(162), 1050-1070.

http://dx.doi.org/10.1590/198053143599

Quintriqueo, S. (2005). Implicancias de la escolarización en la construcción de la identidad cultural de alumnos mapuche en el medio escolar de la IX Región. En D. Quilaqueo, S. Quintriqueo y P. Cárdenas (Eds.), Educación, currículum e interculturalidad. Elementos sobre formación de profesores en contexto mapuche (pp. 191-248). Universidad Católica de Temuco.

Quintriqueo, S. (2010). Implicancias de un modelo curricular monocultural en contexto mapuche. Universidad Católica de Temuco.

Quintriqueo, S. y Quilaqueo, D. (2019). Modelo de Intervención Educativa Intercultural en Contexto Indigena. Editorial Universidad Católica de Temuco.

Quintriqueo, S., Quilaqueo, D., Peña-Cortés, F. y Muñoz, G. (2015). Conocimientos culturales como contenidos de la educación familiar mapuche. A/pha, 40, 131-146. http://dx.doi.org/10.4067/s0718-22012015000100010 Quintriqueo, S., Quilaqueo, D., Lepe-Carrión, P., Riquelme, E., Guitiérrez, M. y Peña-Cortés, F. (2014). Formación del profesorado en educación intercultural en América Latina. El caso de Chile. Revista Electrónica Interuniversitaria de Formación del Profesorado, 172), 201-217. https://doi.org/10.6018/reifop.17.2.198021 Quintriqueo, S., Riquelme, E., Morales, S., Quilaqueo, D. y Gutiérrez, M. (2017). Conocimiento educativo en el contexto escolar y en la educación familiar mapuche: principales tensiones epistemológicas. Revista Brasileira de Educação, 22(71), 1-19. https://www.redalyc.org/pdf/275/27553035011.pdf Quintriqueo, S. y Maheux, G. (2004). Exploración del conocimiento sobre la relación de parentesco como contenido educativo para un currículum escolar intercultural en comunidades mapunche. Revista de Psicología, 13(1), 73-91. https://doi.org/10.5354/0719-0581.2004.17488 
Quintriqueo, S. y Torres, H. (2012). Distancia entre el conocimiento mapuche y el conocimiento escolar en contexto mapuche. Revista Electrónica de Investigación Educativa, 14(1), 16-33.

https://www.redalyc.org/pdf/155/15523175002.pdf

Salas, S., Godino, J. y Quintriqueo, S. (2016). Análisis Exploratorio de las Prácticas Matemáticas de dos

Estudiantes Mapuches en Colegios con y sin Educación Intercultural Bilingüe. Journal BOLEMA: boletim de Educação Matemática. http://dx.doi.org/10.1590/1980-4415v30n55a09

Valdebenito, X. (2017). Hacia una comprensión del vínculo entre las prácticas de enseñanza de educadores y educadoras tradicionales indigenas y el espacio escolar. Centro de estudios MINEDUC. 